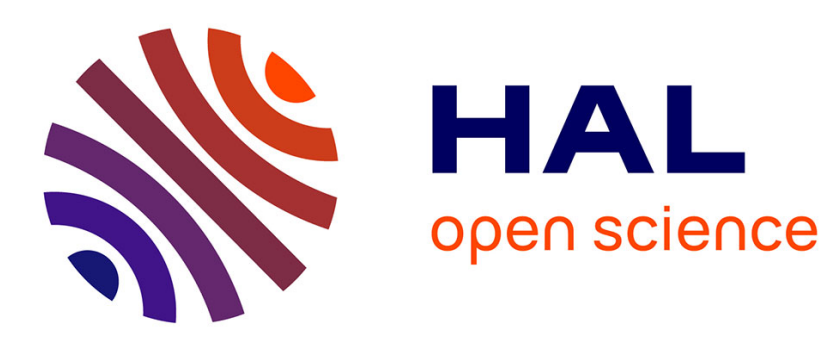

\title{
Information Extraction by Blind Source Separation from Polarimetric SAR Data
}

Leandro Pralon, Gabriel Vasile, Mauro Dalla Mura, Jocelyn Chanussot

\section{To cite this version:}

Leandro Pralon, Gabriel Vasile, Mauro Dalla Mura, Jocelyn Chanussot. Information Extraction by Blind Source Separation from Polarimetric SAR Data. IGARSS 2017 - IEEE International Geoscience and Remote Sensing Symposium, Jul 2017, Fort Worth, United States. hal-01593486

\section{HAL Id: hal-01593486 \\ https://hal.science/hal-01593486}

Submitted on 26 Sep 2017

HAL is a multi-disciplinary open access archive for the deposit and dissemination of scientific research documents, whether they are published or not. The documents may come from teaching and research institutions in France or abroad, or from public or private research centers.
L'archive ouverte pluridisciplinaire HAL, est destinée au dépôt et à la diffusion de documents scientifiques de niveau recherche, publiés ou non, émanant des établissements d'enseignement et de recherche français ou étrangers, des laboratoires publics ou privés. 


\title{
INFORMATION EXTRACTION BY BLIND SOURCE SEPARATION FROM POLARIMETRIC SAR DATA
}

\author{
Leandro Pralon ${ }^{* * *}$, Gabriel Vasile*, Mauro Dalla-Mura*, Jocelyn Chanussot ${ }^{*}$ \\ * Grenoble-Image-sPeach-Signal-Automatics Lab, GIPSA-lab \\ Univ. Grenoble Alpes, CNRS / Grenoble-INP, Grenoble, France \\ \{gabriel.vasile $\|$ mauro.dalla-mura ||jocelyn.chanussot\}@ gipsa-lab.grenoble-inp.fr \\ ** Brazilian Army Technological Center \\ Rio de Janeiro-RJ 23020-470, Brazil \\ pralon@ctex.eb.br
}

\begin{abstract}
Cloude and Pottier $\mathrm{H} / \alpha$ feature space [1] is one of the most employed methods for unsupervised PolSAR data classification based on Incoherent Target Decomposition. The association of the coherence matrix eigenvectors to the most dominant scatters in the analysed pixel introduces unfeasible regions in the $\mathrm{H} / \alpha$ plane. The Independent Component Analysis provides promising new information to better interpret non-Gaussian heterogeneous clutter in the frame of polarimetric incoherent target decompositions. Not constrained to any orthogonality between the estimated scattering mechanisms that compose the clutter under analysis, ICA does not introduce any unfeasible region in the $\mathrm{H} / \alpha$ plane, increasing the range of possible natural phenomenons depicted in the aforementioned feature space.
\end{abstract}

Index Terms - Independent Component Analysis

\section{INTRODUCTION}

Polarimetric target decomposition is a PolSAR image interpretation technique that relies on the analysis of the interaction between the illuminated area and the transmitted waveform, considering each polarimetric state of the latter. Compared to the univariate analysis of single polarization systems, the multivariate nature of PolSAR data allows for a better prediction of the physical properties of the illuminated targets, leading to more effective classification, detection and geophysical parameter inversion algorithms. More specifically, with respect to target decompositions, they enable the description of an image cell as a sum of canonical scattering mechanisms (also called as target vectors) making it more intuitive to understand the behaviour of the clutter and therefore to analyse it [2].

Polarimetric target decompositions are mainly classified in coherent, if their interest lies on the scattering matrix

Thanks to the DLR for providing the Mont Blanc PolSAR data. analysis for each resolution cell, like the ones proposed by Cameron [3], or incoherent, if they are based on a statistical analysis of neighbouring pixels. Incoherent target decomposition (ICTD) theory assumes that the scattering process in most natural media is a combination of coherent speckle noise and random vector scattering effects. Therefore, a stochastic approach is required and the concept of average or dominant scattering mechanisms is associated to each imaging cell [4]. Most methods described in the literature focus on the Hermitian, semidefinite positive coherence or covariance matrix [4]. Nevertheless, the investigation of higher order moments has recently sparked great interest of the SAR community, introducing supplementary information to the clutter analysis and consequently leading to new ICTD approaches [5], [6], [7].

ICTD algorithms can be split in two stages: the decomposition of an image pixel into basic target vectors and the correct retrieval of quantitative information from them (parametrization). Concerning the latter, Cloude and Pottier's parameters (entropy, alpha and anisotropy) [1] and Touzi's target scattering vector model [2] are the most employed ones, whose usefulness have already been demonstrated by several authors. Regarding the former, the association of the three most dominant scatters in an image cell to the eigenvectors of the coherence/covariance matrix of the data is so widespread in the PolSAR community that it is often mistaken as the only alternative for that purpose. The Eigenvector based ICTD has an intrinsic property that the derived scatters are orthogonal and uncorrelated, which for Gaussian clutters also means independence. The drawback of this kind of method emerge when the clutter is not Gaussian or not composed by orthogonal mechanisms, situations where the performance of the algorithm could be compromised.

The combined use of the Eigenvector approach with Cloude and Pottier's parametrization gave rise to one of the most employed and most traditional classification schemes in PolSAR data analysis, the $\mathrm{H} / \alpha$ feature space [1]. The en- 
tropy, $\mathrm{H}$, measures the degree of randomness of the scattering phenomenon, given as a function of the eigenvalues of the coherence matrix. Low entropy values indicate that a dominant scatter is mainly responsible for the given polarimetric signature while high values (close to 1 ) point to the existence of multiple scattering mechanisms within the image cell, suggesting that there is not a single one that stands out among the others. The $\alpha$ angle is extracted from the eigenvectors of the coherence matrix and represents the physical behaviour of the scatter, ranging from an isotropic surface $\left(\alpha=0^{0}\right)$ to double bounce type of targets $\left(\alpha=90^{\circ}\right)$, passing trough oriented dipole $\left(\alpha=45^{0}\right)$. Each eigenvector correspond to a scattering mechanism within the image cell and therefore each one will provide a different $\alpha$ angle. The authors in [1] state that the best estimate of such parameter to represent the image cell is an weighted average based on the eigenvalues of the coherence matrix.

The parameters $\mathrm{H}$ and $\alpha$ are plotted in a plane, originating the so called $\mathrm{H} / \alpha$ feature space. Upon the introduction of the aforementioned method, Cloude and Pottier suggested the partitioning of the plane in 9 regions, based on the polarimetric behaviour of known type of natural phenomenons. Therefore, once the $\mathrm{H}$ and the average $\alpha$ parameters are extracted from the target polarimetric signature, it is straightforward to classify it as one of the corresponding type of scattering mechanisms. Many works are based on such method, from geophysical parameters inversion algorithms (in varied regions from the globe) to detection and classification algorithms. Having a remarkable correspondence to ground truth, the usage of this unsupervised technique has had very few improvements since its conception. Nevertheless, there is an important remark still uncovered related to this method. The orthogonality constraint of the eigenvectors of the coherence matrix generates unfeasible regions in the plane. It is important to highlight that these regions are mathematically, and not physically, unfeasible.

Within this context, this paper addresses the potential new information provided by the ICA as an ICTD method with respect to Cloude and Pottier $\mathrm{H} / \alpha$ feature space.

\section{INDEPENDENT COMPONENT ANALYSIS ICTD}

The limiting curves on the $\mathrm{H} / \alpha$ plane reduce the analysis of high entropy type of targets in a more limiting way than the analysis of low entropy ones. Even though polarimetry is a better suited tool to analyse low entropy type of targets, the ability to correctly estimate the parameters that describe the illuminated scatters is crucial. Therefore, any constraint that prevent this from happening decreases the performance of any classification, detection and geophysical parameter inversion algorithms. Furthermore, when surface type of scatters are addressed, even in low entropy environment the constraint on the estimated average $\alpha$ angle can lead to a misinterpretation of the actual behaviour of the clutter, directly affecting the performance of classification and detection algorithms as well as the validity of many physical models widely used to describe this type of targets.

Another evidence that the orthogonality constraint introduced by the Eigenvector approach may compromise the performance of classification algorithms arises when the $\mathrm{H} / \alpha$ feature space is used as a starting point in iterative classification algorithms. Iterative classification algorithms, like the ones proposed in [8], rely on the assumption that pixels with similar statistical behaviour may compose a same cluster (class) even if they reside in different boundaries regions. Therefore, once an initial population is defined (generally the output of the $\mathrm{H} / \alpha$ classification), a distance measure based on the stochastic model adopted is taken into consideration to verify if a given pixel is more likely to be part of a new class than its actual one. In many applications it is noticeable the migration of pixels originally classified in zone 6 to zone 2 , indicating that pixels in these regions have characteristics that are not well described by neither of phenomenons represented by them.

In [7], a new strategy to polarimetric target decomposition was presented by incorporating the independent component analysis (ICA) as an alternative to identify the canonical scattering mechanisms within an image cell. The ICA is a blind source separation technique based on higher order statistical moments and cumulants whose utility has already been explored in many different research areas, such as wireless communications, data compression and brain imaging applications. The results presented in [7] proved it to be a very promising area in polarimetry, mainly when nonGaussian heterogeneous clutters (inherent to high resolution SAR systems) are under study. The theoretical potential in estimating similar entropy and first component, when compared to traditional eigenvector decomposition, but rather a second most dominant component independent with respect to the first one and unconstrained by the orthogonality introduces an alternative way of physically interpreting a polarimetric SAR image. In [9], the ability of ICA to correctly identify clutters composed by non-orthogonal type of scatters is further investigated, as well as its performance under a sliding window approach, enabling a more accurate comparison to the results obtained with the Eigenvector based approach.

In general, the limiting curves on the $\mathrm{H} / \alpha$ plane reduce the analysis of high entropy type of targets in a more limiting way than the analysis of low entropy ones. Even though polarimetry is a better suited tool to analyse low entropy type of targets, the ability to correctly estimate the parameters that describe the illuminated scatters is crucial. Once again, it is important to highlight that these regions are mathematically, and not physically, unfeasible.

In the present work, our focus relies on the analysis of pixels whose polarimetric behaviour falls into regions 2,3 and 6 . According to the authors in [2], region 6 represents a medium entropy surface scatter. When compared to the expected be- 
haviour of surface mechanisms, it is stated that the increase in entropy can be related to either an increase in the surface roughness or due to canopy propagation effects. If the entropy increased further and the $\alpha$ range was kept unchanged, pixels would fall in region 3 and would be classified as a high entropy surface. Nevertheless, due to the mathematical constraint discussed before, no scattering mechanisms can be identified as belonging to this region. Finally, region 2, stands for high entropy vegetation scattering, including volume type of scatters. The natural behaviour of forest canopies and some types of vegetated surfaces with random highly anisotropic scattering elements may fall in this region [2]. Note that the physical phenomenologies represented by region 6 and region 2 are very different. Therefore, it should be expected very few doubt in determining if a pixel is better represented by region 6 or by region 2 .

\section{POLSAR EXPERIMENTAL RESULTS}

The PolSAR dataset was acquired in October 2006 by the ESAR system over the upper part of the Tacul glacier from the Chamonix - Mont Blanc test site, France. Figure 1 presents the referred area in Red $(\mathrm{HH}+\mathrm{VV})$, Green $(\mathrm{HV})$ and Blue (HH-VV) and the output of the Spherical Symmetry test [10] where in green are represented Non Spherical Symmetric pixels. The latter describes the degree of heterogeneity of the investigated scene.

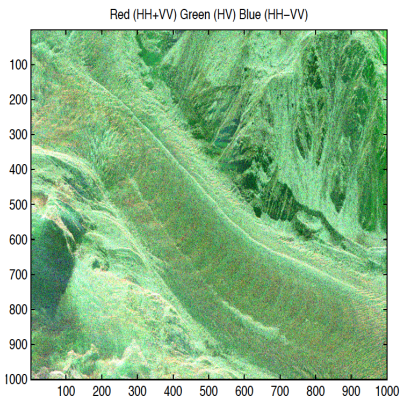

(a)

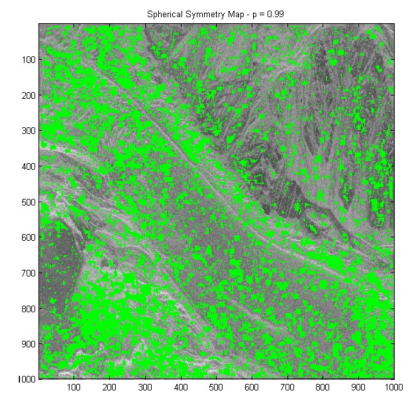

(b)
Fig. 1. Tacul glacier area under study: (a) RGB image, Red (HH+VV), Green (HV), Blue (HH-VV); (b) Spherical symmetry map.

Note that only a small portion of the pixels in the area under study fails the test (29\%), indicating that the SIRV model is also a good representative of this dataset stochastic behaviour. Figure 2 presents the initial $\mathrm{H} / \alpha$ classification output for both Eigenvector approach and ICA based ICTD, the statistical classification algorithm [11] output after 4 iterations. The corresponding $\mathrm{H} / \alpha$ feature spaces are depicted in 3 .

The amount of pixels that move from class 6 to class 2 when the Eigenvector approach is employed represents just $34.70 \%$ of the set originally classified in region 6 . Nevertheless, even though the displacement of pixels between classes 6

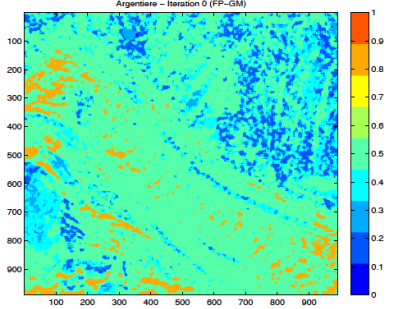

(a)

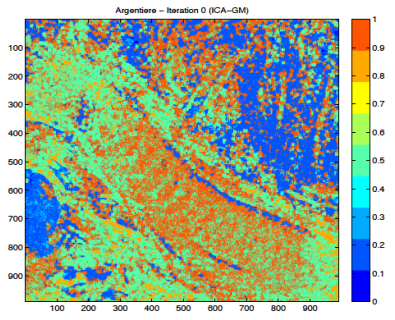

(c)

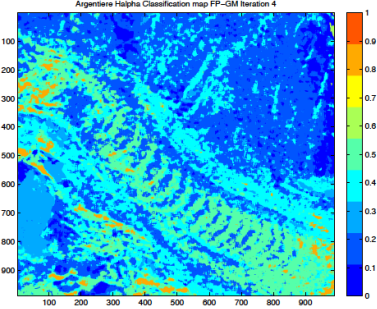

(b)

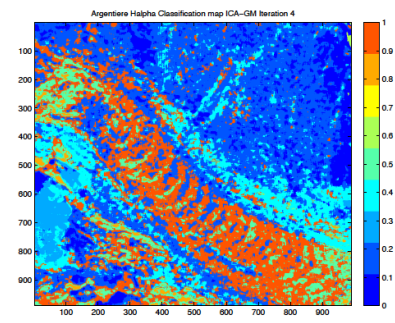

(d)
Fig. 2. Tacul glacier area under study: (a) $\mathrm{H} / \alpha$ classification output Eigenvector approach; (b) Statistical classification algorithm output, 4th iteration Eigenvector approach; (c) $\mathrm{H} / \alpha$ classification output ICA based ICTD; (d) Statistical classification algorithm output, 4th iteration ICA based ICTD.

and 2 is negligible, scatters represented by region 3 in the $\mathrm{H} / \alpha$ feature space not only are still present but also are well clustered, specially after the employment of the statistical classification algorithm. The latter is an evidence that region 3 may be indicating a specific natural phenomenon unable to be represented when the Eigenvector approach is taken into consideration.

Indeed, region 3 is mainly characterizing the pixels that represent the curved stripes due to the Forbess bands phenomena, a periodical feature caused by a regularly recurrent displacement of the Tacul glacier during a year. The Forbess band can be identified in Figure 6.8 as the succession of undulations which sweeps transversely across the sensed region.

In [12], the authors interpreted the corresponding scattering mechanisms as a dominant surface backscatter with low entropy and alpha angles. This is in accordance to what is depicted in Figure 6.8 (see also Figure 6.9). Nevertheless, note that a considerable portion of pixels in this area correspond to class 4 which does not, in theory, explain the physical phenomenon. The ICA approach on the other hand indicates that the aforementioned region is initially better characterized by a high entropy surface backscattering signature, which is unfeasible in the Eigenvector based ICTD using Cloude and Pottier parametrization. Whilst the typical alpha angles estimated using both methods are nearly the same, the entropy derived with ICA is higher, and the existence of pixels characterized as belonging to class 4 is negligible. If no classification algorithm was employed, it would be already possible to characterize the dirty bands and estimate geophysical param- 


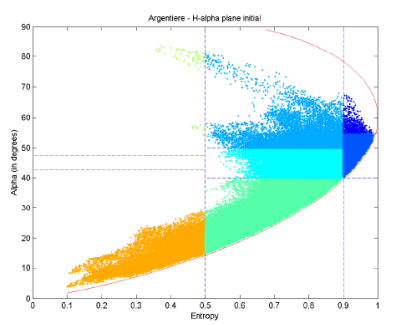

(a)

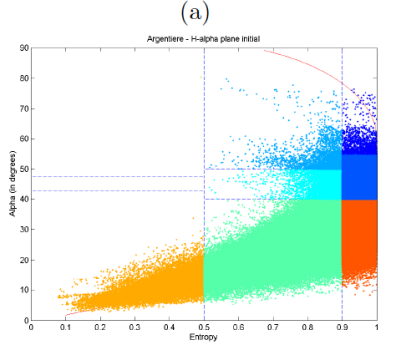

(c)

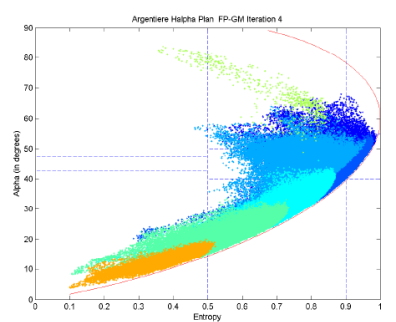

(b)

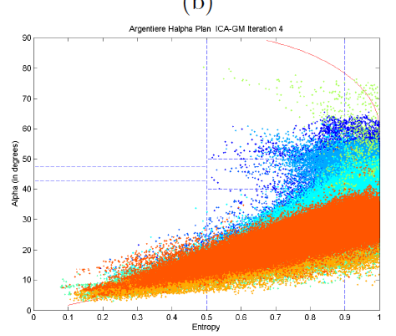

(d)
Fig. 3. Tacul glacier area under study: (a) $\mathrm{H} / \alpha$ initial feature space Eigenvector approach; (b) $\mathrm{H} / \alpha$ feature space after the 4th iteration Eigenvector aproach; (c) $\mathrm{H} / \alpha$ initial feature space ICA based ICTD; (d) $\mathrm{H} / \alpha$ feature space after the 4 th iteration ICA based ICTD.

eters related to them taking into consideration only the initial population derived with the ICA based ICTD (using a sliding window approach).

Among other improvements, the statistical classification output clearly delimits the aforementioned regions, increasing the ability to better characterize them. The correct identification of Furthermore, note that the expected behaviour of the dirt bands as they approach the bottoms, is met in the ICA based approach, i.e., they are reduced as the glacier descends until they disappear, which can be observed by the higher amount of pixels classified in class 5 (medium entropy surface) in the bottom of the glacier.

\section{CONCLUSION}

This paper addressed the potential new information provided by ICA based ICTD in terms of Cloude and Pottier $\mathrm{H} / \alpha$ feature space. A theoretical analysis of the limiting curves described in [1] that generate unfeasible regions in the aforementioned plane show that they arise as a consequence of the orthogonality constraint inherent to the coherence matrix eigenvectors, which are associated to the most dominant scatters present in the analysed pixel. Since the ICA based ICTD does not introduce such constraint, examples of possible clutter compositions with non-orthogonal mechanisms that fall outside the Eigenvector approach feasible regions were addressed.

\section{REFERENCES}

[1] S. R. Cloude and E. Pottier, "An entropy based classification scheme for land applications of polarimetric SAR," IEEE Transactions on Geoscience and Remote Sensing, vol. 35, no. 1, pp. 68-78, 1997.

[2] R. Touzi, "Target scattering decomposition in terms of rollinvariant target parameters," IEEE Transactions on Geoscience and Remote Sensing, vol. 45, no. 1, pp. 73-84, 2007.

[3] W. L. Cameron and L. K. Leung, "Feature motivated polarization scattering matrix decomposition," in Proceedings of the IEEE International Radar Conference, Arlington, USA, 1990, pp. 549-557.

[4] S. R. Cloude and E. Pottier, "A review of target decomposition theorems in radar polarimetry," IEEE Transactions on Geoscience and Remote Sensing, vol. 32, no. 6, pp. 498-518, 1996.

[5] G. Vasile, Imagerie radar à synthse d'ouverture interférométrique et polarimétrique. Application au suivi des glaciers alpins, Ph.D. thesis, Université de Savoie, France, 2007.

[6] G. Vasile, F. Pascal, J.-P. Ovarlez, P. Formont, and M. Gay, "Optimal parameter estimation in heterogeneous clutter for high resolution polarimetric SAR data," IEEE Geoscience and Remote Sensing Letters, vol. 8, no. 6, pp. 1046-1050, 2011.

[7] N. Besic, G. Vasile, J. Chanussot, and S. Stankovic, "Polarimetric incoherent target decomposition by means of independent component analysis," IEEE Transactions on Geoscience and Remote Sensing, vol. 53, no. 3, pp. 1236-1247, 2015.

[8] J. S. Lee, M. R. Grunes, T. L. Ainsworth, D. Li-Jen, D. L. Schuler, and S. R. Cloude, "Unsupervised classification using polarimetric decomposition and the complex Wishart classifier," IEEE Transactions on Geoscience and Remote Sensing, vol. 37, no. 5, pp. 2249-2258, 1999.

[9] L. Pralon, G. Vasile, M. Dalla-Mura, J. Chanussot, and N. Besic, "Evaluation of ICA-based ICTD for PolSAR data analysis using a sliding window approach: convergence rate, gaussian sources, and spatial correlation," IEEE Transactions on Geoscience and Remote Sensing, vol. 54, no. 7, pp. 42624271, 2016.

[10] L. Pralon, G. Vasile, M. Dalla-Mura, A. Anghel, and J. Chanussot, "Spherical symmetry of complex stochastic models in multivariate high-resolution PolSAR images," IEEE Transactions on Geoscience and Remote Sensing, vol. 54, no. 7, pp. 4250-4261, 2016.

[11] P. Formont, F. Pascal, G. Vasile, J.-P. Ovarlez, and L. FerroFamil, "Statistical classification for heterogeneous polarimetric SAR images," IEEE Journal of Selected Topics in Signal Processing, vol. 5, no. 3, pp. 398-407, 2011.

[12] T. Landes, M. Gay, E. Trouve, J.M Nicolas, L. Bombrun, G. Vasile, and I. Hajnsek, "Monitoring temperate glaciers by high resolution Pol-InSAR data: First analysis of Argentiere E-SAR acquisitions and in-situ measurements," in Proceedings of the IEEE International Geoscience and Remote Sensing Symposium, Cape Town, South Africa, 2007, pp. 184-187. 